\title{
Clinical utility of chromosomal microarray analysis to detect copy number variants: Experience in a single tertiary hospital
}

\author{
Hee Sue Park ${ }^{1} * \oplus$, Aryun $\mathrm{Kim}^{2 \oplus}$, Kyeong Seob Shin ${ }^{1}$, and Bo Ra Son ${ }^{1 \oplus}$ \\ ${ }^{1}$ Department of Laboratory Medicine, Chungbuk National University Hospital, Chungbuk National University College of Medicine, Cheongju, Korea \\ ${ }^{2}$ Department of Neurology, Chungbuk National University Hospital, Chungbuk National University College of Medicine, Cheongju, Korea
}

\begin{abstract}
Purpose: To summarize the results of chromosomal microarray analysis (CMA) for copy number variants (CNVs) detection and clinical utility in a single tertiary hospital.

Materials and Methods: We performed CMA in 46 patients over the course of two years. Detected CNVs were classified into five categories according to the American College of Medical Genetics and Genomics guidelines and correlated with clinical manifestations.

Results: A total of $31 \mathrm{CNVs}$ were detected in 19 patients, with a median CNV number per patient of two CNVs. Among these, 16 CNVs were classified as pathogenic $(n=3)$ or likely pathogenic $(L P)(n=11)$ or variant of uncertain significance $(n=4)$. The 16p11.2 deletion and 16p13.11 deletion classified as LP were most often detected in 6.5\% (3/46), retrospectively. CMA diagnostic yield was $24.3 \%$ (9/37 patients) for symptomatic patients. The CNVs results of the commercial newborn screening test using next generation sequencing platforms showed high concordance with CMA results.

Conclusion: CMA seems useful as a first-tier test for developmental delay with or without congenital anomalies. However, the classification and interpretation of CMA still remained a challenge. Further research is needed for evidence-based interpretation.
\end{abstract}

Key words: Chromosome microarray analysis, Copy number variations, Developmental disability, Genetic testing, Highthroughput nucleotide sequencing.

\section{Introduction}

Genetic diseases are caused by genomic aberrations in single nucleotide variations leading to structural changes including deletion, duplication, and translocations. G-banded chromosome analysis is a standard method to detect chromosome abnormalities, but it is limited in its sensitivity, since it only detects structure abnormalities $\geq 3-10 \mathrm{Mb}[1]$. On the other hand, chromosomal microarray analysis (CMA) is an excellent method for detecting microdeletions or duplications $<400 \mathrm{~kb}$ [2]. In addition, CMA can be more useful because the interpretation of Gbanded assay depends on the examiner's experience whereas

\footnotetext{
Received: 29 April 2021, Revised: 10 June 2021, Accepted: 13 June 2021, Published: 30 June 2021

${ }^{*}$ Corresponding author: Hee Sue Park, M.D., Ph.D. (D) https://orcid.org/0000-0002-8378-6066

Department of Laboratory Medicine, Chungbuk National University Hospital, Chungbuk National University College of Medicine, 776, 1sunhwan-ro, Seowon-gu, Cheongju 28644, Korea.

Tel: +82-43-269-7345, Fax: +82-43-271-5243, E-mail: pheno27@naver.com

Conflict of interest: The authors declare that they do not have any conflicts of interest.

(ac) This is an open-access article distributed under the terms of the Creative Commons Attribution Non-Commercial License (http://creativecommons.org/licenses/by-nc/4.0/) which permits unrestricted non-commercial use, distribution, and reproduction in any medium, provided the original work is properly cited.

(c) Copyright 2021 by the Korean Society of Medical Genetics and Genomics 
Table 1. Clinical characteristics of 37 patients with clinical features

\begin{tabular}{|c|c|c|c|c|c|}
\hline Patient no. & Age & Sex & Clinical symptoms & Congenital anomaly & Familial history \\
\hline S1 & $8 \mathrm{D}$ & $\mathrm{M}$ & Developmental delay, epilepsy & Cleft palate & - \\
\hline S2 & $2 \mathrm{M}$ & M & Epilepsy & Cortical malformation & - \\
\hline S3 & $3 Y$ & $\mathrm{~F}$ & Developmental delay & Horseshoe kidney & Febrile seizure in aunt \\
\hline S4 & $14 \mathrm{D}$ & F & Hypotonia & - & - \\
\hline S5 & $3 Y$ & M & Developmental delay & - & - \\
\hline S6 & $8 Y$ & M & Developmental delay & - & - \\
\hline S7 & $5 Y$ & M & Developmental delay & Facial dysmorphism & - \\
\hline S8 & $25 \mathrm{D}$ & $\mathrm{F}$ & Developmental delay & Aniridia, macrocephaly & Aniridia in father \\
\hline S9 & $27 \mathrm{D}$ & $\mathrm{F}$ & $\begin{array}{l}\text { Epilepsy, small for gestational age, } \\
\text { respiratory distress }\end{array}$ & Hemivertebra & - \\
\hline $\mathrm{S} 10$ & $22 \mathrm{D}$ & M & Developmental delay, epilepsy & - & - \\
\hline S11 & $20 \mathrm{D}$ & M & Developmental delay & - & - \\
\hline S12 & $5 \mathrm{M}$ & $\mathrm{F}$ & Developmental delay, epilepsy & - & - \\
\hline $\mathrm{S} 13$ & $9 \mathrm{M}$ & $\mathrm{F}$ & Developmental delay & - & Low height in brother \\
\hline $\mathrm{S} 14$ & $5 Y$ & M & Developmental delay & $\begin{array}{l}\text { Microcephaly, } \\
\text { retinal degeneration }\end{array}$ & Visual impairment in mother \\
\hline S15 & $4 Y$ & M & Developmental delay, epilepsy & - & Seizure in aunt \\
\hline S16 & $3 Y$ & M & & Flat feet & Club foot in brother \\
\hline S17 & $12 Y$ & $\mathrm{~F}$ & Asthma & Pulmonary agenesis & - \\
\hline $\mathrm{S} 18$ & $4 Y$ & $\mathrm{~F}$ & Developmental delay & Strabismus & Strabismus in uncle \\
\hline S19 & $4 Y$ & M & Developmental delay & - & - \\
\hline S20 & $2 Y$ & M & Developmental delay & Dysmorphic face & - \\
\hline $\mathrm{S} 21$ & $5 Y$ & M & Developmental delay & - & - \\
\hline $\mathrm{S} 22$ & $2 Y$ & M & Developmental delay & - & - \\
\hline $\mathrm{S} 23$ & $12 Y$ & M & Developmental delay, epilepsy & - & - \\
\hline S24 & $5 Y$ & M & Developmental delay, epilepsy & - & Febrile seizure in father \\
\hline S25 & $35 Y$ & $\mathrm{~F}$ & Recurrent abortion & - & - \\
\hline S26 & $0 \mathrm{M}$ & M & $\begin{array}{l}\text { Low birth weight, hypothyroidism, } \\
\text { hypocalcemia }\end{array}$ & - & - \\
\hline S27 & $1 Y$ & M & Developmental delay, epilepsy & Dolicocephaly, nystagmus & - \\
\hline S28 & $18 Y$ & M & Common variable immunodeficiencies & - & - \\
\hline S29 & $1 Y$ & M & Developmental delay, epilepsy & - & - \\
\hline S30 & $4 \mathrm{M}$ & M & $\begin{array}{l}\text { Nephrogenic diabetes insipidus, } \\
\text { developmental delay }\end{array}$ & - & - \\
\hline S31 & $2 Y$ & M & Developmental delay, epilepsy & $\begin{array}{l}\text { Facial dysmorphism, } \\
\text { macrocephaly }\end{array}$ & - \\
\hline S32 & $2 Y$ & M & Developmental delay & - & - \\
\hline S33 & $1 \mathrm{M}$ & M & Epilepsy & Hydranencephaly & - \\
\hline S34 & $3 Y$ & M & Developmental delay & - & - \\
\hline S35 & $3 Y$ & M & Epilepsy & Atrial septal defect & - \\
\hline S36 & $18 \mathrm{M}$ & $\mathrm{F}$ & Developmental delay & - & - \\
\hline S37 & $13 Y$ & $\mathrm{~F}$ & Developmental delay & - & Developmental delay in father \\
\hline
\end{tabular}

D, day; $M$, month; $Y$, year; $M$, male; F, female; -, not remarkable.

CMA does not. Therefore CMA is a primary approach for copy number variant (CNVs) detection [3], whose clinical usefulness has been reported in clinical practices aimed to detect specific developmental delays [4].

However, CNVs is common in the healthy population and reported in about 35\% of people [2,5]. By 2015, over 2.3 million
CNVs were reported in the database of genomic variants [6], and the number of CNVs is expected to increase further with the commercialization of new CMA and next-generation sequencing (NGS) techniques. Therefore, an accurate interpretation of variants is required, and it is important to evaluate laboratory methods and correlation with clinical phenotypes [7]. To deter- 


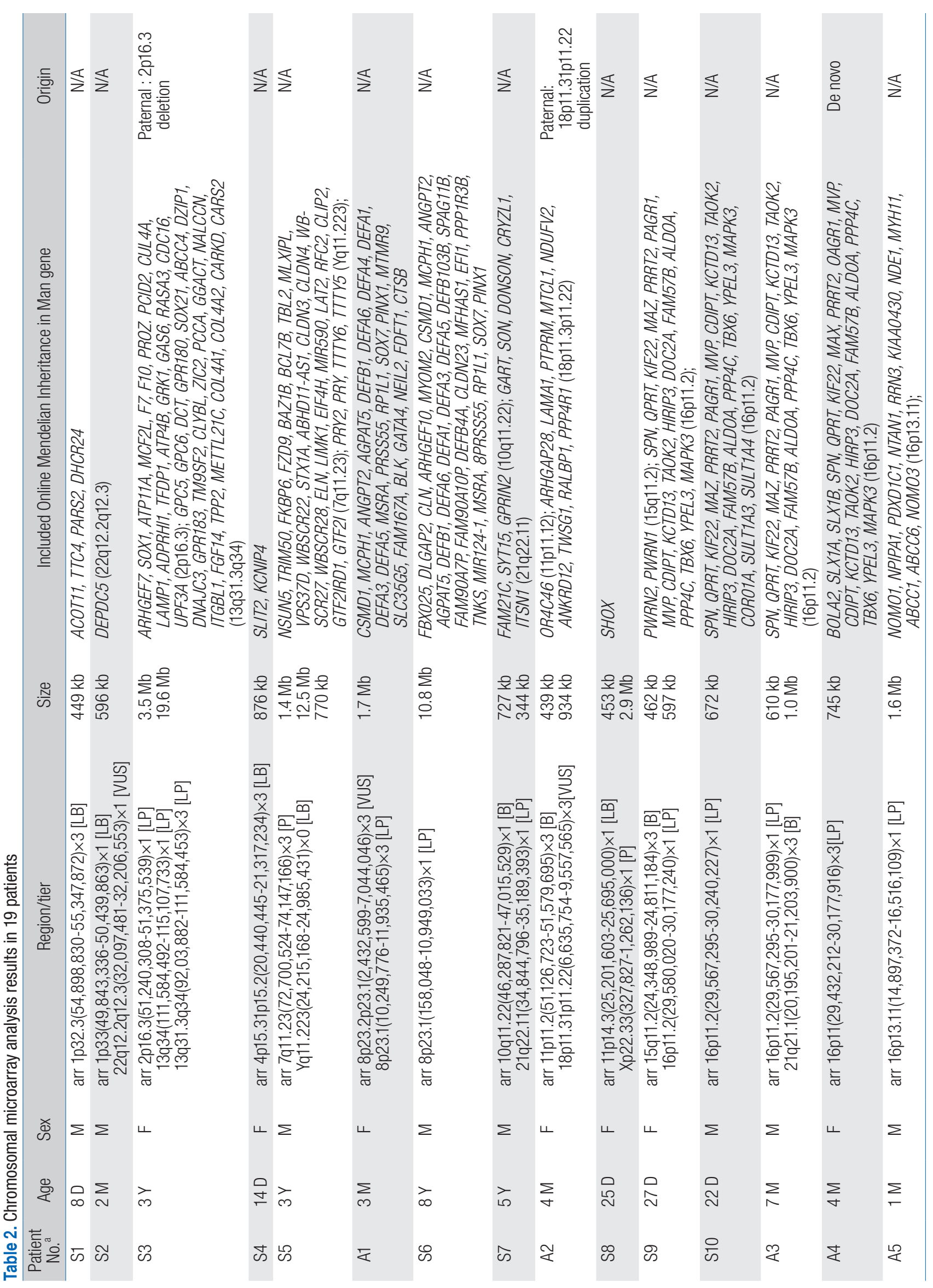




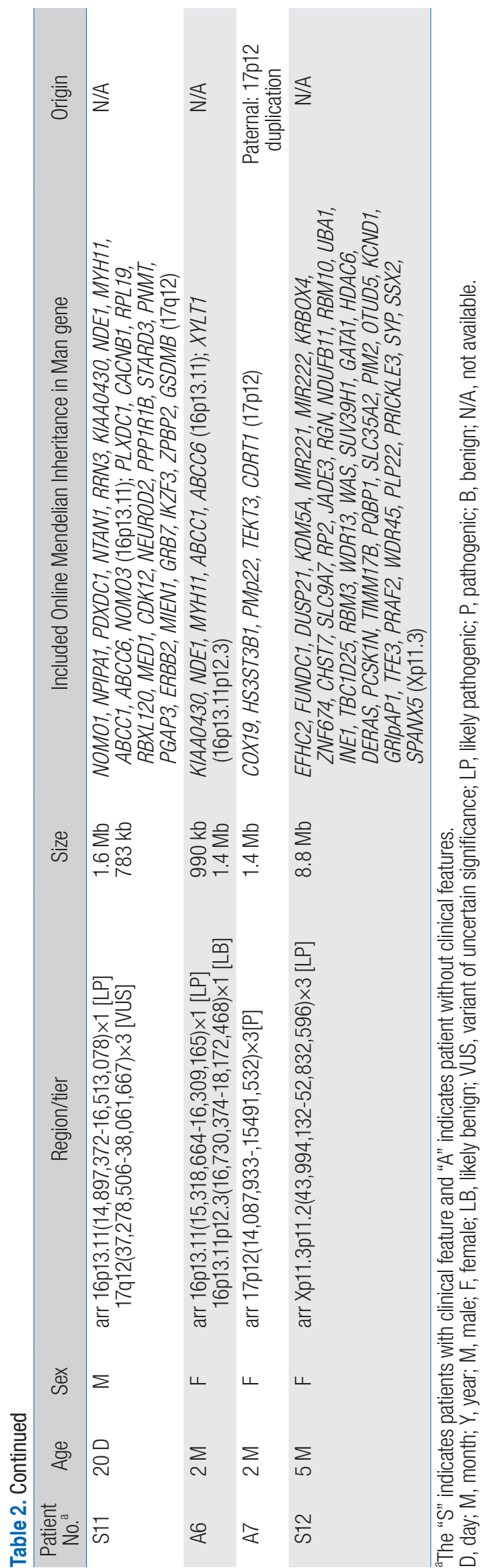

mine the classification categories of CNVs, preexisting published literatures and population database according to ethnic groups are valuable evidence [7], so data sharing is a must in this field of research. This study aims to summarize the results of CMA for CNV detection in a single institution and evaluate its clinical usefulness.

\section{Materials and Methods}

This study included 46 patients for whom CMA was requested at the Chungbuk National University Hospital between 2019 and 2020. Peripheral blood samples were collected in ethylenediaminetetraacetic acid tubes. All procedures were performed at Green Cross Laboratories (Yongin, Korea) and performed with CytoScan Dx Assay (Affymetrix Inc., Santa Clara, CA, USA). Human Genome Build 19 was used to perform the description of genomic variants. CNVs were classified into five categories according to the American College of Medical Genetics and Genomics guidelines into pathogenic (P), likely pathogenic (LP), uncertain significance (VUS), likely benign (LB) and benign (B) [7]. This study was approved by the institutional review board of Chungbuk National University Hospital (IRB number: CBNU2021-03-030).

\section{Results}

Of the 46 patients for whom CMA was requested, 37 had clinical symptoms (patient number S1-S37), among which developmental delay was the most common clinical feature (Table 1). The other 9 patients (patient number A1-A9) were requested based on findings on commercial CNVs neonatal screening test using NGS methods.

A total of 31 CNVs were detected in 19 patients as in Table 2. The median detected indel region size was $934 \mathrm{~kb}$ ( $\min 344 \mathrm{~kb}$ max $19.6 \mathrm{Mb}$ ), and the median number of detected CNVs per patient was 2 ( $\min 1-\max 3)$. The CNVs were classified as $P(n=3)$, $L P(n=14), \operatorname{VUS}(n=4), L B(n=6)$, and $B(n=4)$. Excluding duplicates, there were $17 \mathrm{CNV}$ s classified as $P(n=3)$ or $L P(n=10)$ or VUS ( $n=4)$ (Table 3). The 16p11.2 deletion and 16p13.11 deletion classified as likely LP were most often detected in 6.5\% (3/46), retrospectively. In particular, the $16 p 11.2$ deletion appears with high frequency in several reports regardless of ethnic, but the $16 p 13.11$ deletion is relatively rare $[5,8]$. In contrast, $8 p 23.1$ region seems to be a more recurrent region in Korea.

Of all 37 symptomatic patients, 12 patients had one more CNVs. Detected CNVs were classified as $P(n=2), L P(n=9)$, and 


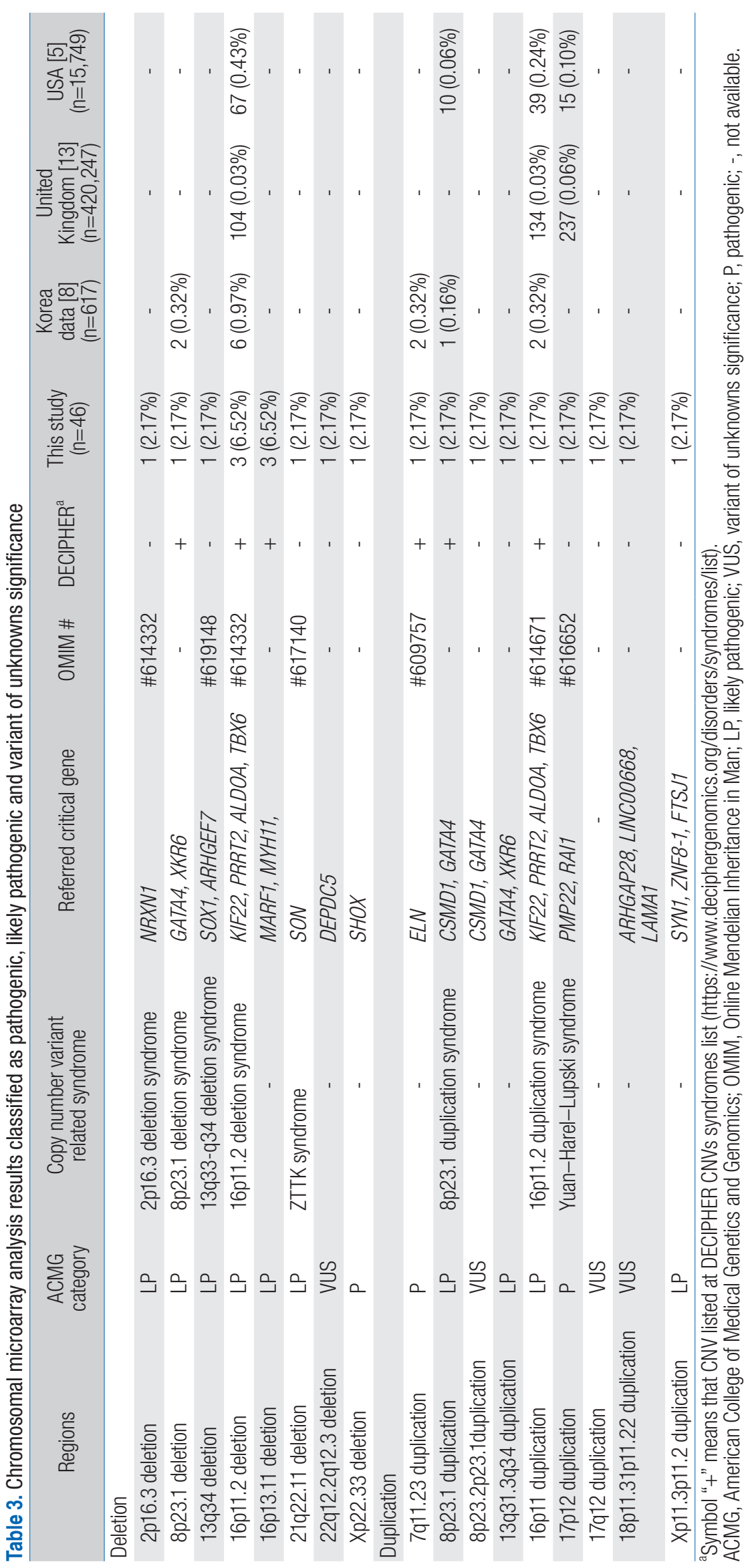


VUS $(n=2)$ in 10 patients, while 7 LB CNVs were observed in seven patients. In addition, the remaining 25 patients had a normal karyotype without any CNVs. In the asymptomatic group $(n=9)$, detected CNVs were classified as $P(n=6), B(n=2)$, and VUS $(n=1)$ (Fig. 1). Five of the 27 patients with normal CMA findings underwent an additional targeted NGS panel of genes associated with clinical manifestations, but only in three patients additional VUS missense variant were detected. Therefore, it is worth considering follow-up observation or extended whole exome tests for such patients.

\section{Discussion}

The various clinical manifestations depend on genetic defects; therefore, it is very important to select appropriate diagnostic tools to detect the underlying genetic abnormalities. Recently, several reports have shown it is possible to detect CNV even with NGS, as its detection limit is $<300 \mathrm{~kb}$, with a diagnostic sensitivity increasing in proportion to size variations $[9,10]$. In our study, the result of the newborn screening test using NGS platforms showed high concordance with CMA results, except for two discrepancy cases which showed abnormal findings in NGS but normal in CMA. These CNVs were also actually detected in CMA, but not reported in accordance with our institution's the reporting protocol, which excludes benign CNV less than $400 \mathrm{~kb}$. One case had a suspicious finding of $9 q 24.3$ region $73 \mathrm{~kb}$ deletion in NGS, and another case had a suspicious finding of 17p13.3p13.1 region $366 \mathrm{~kb}$ duplication in NGS. Therefore, NGS platforms for CNV detection appear to be cost effective and useful as screening test. Nevertheless, because NGS can only detect specific targeted regions, CMA is more comprehensive for all genomic CNVs. In addition, the break point can be confirmed with CMA clearly.

Similar to previous reports, CMA's diagnostic yield was 24.3\% (9/37) for patients with matching P/LP CNVs and clinical symptoms. Most of these patients (S3, S5, S6, S7, S8, S10, S12, and S13) showed global developmental delay, thus confirming CMA's usefulness in the diagnosis of patients with developmental delay. However, in 27 out of 37 symptomatic patients showed normal results in CMA. Although CMA improves diagnostic yield, there are still undiagnosed cases. Consequently, further clinical management guidelines are needed. If a meaningful result is not

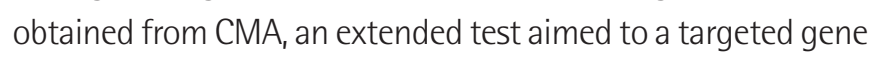
panel, whole exome, or whole genome sequencing should be attempted, while the possibility of an inborn metabolic error also should be discussed [11].

As previously known, there was a different effect of deletions or duplications in the same chromosomal position leading to phenotypic differences in clinical expression. In the case of the chromosome 16p11.2, the most frequently detected in our study, although autism spectrum disorder and neurodevelopmental disorder were common clinical manifestation in both deletion and duplication, speech articulation abnormalities and limb and trunk hypotonia were more reported in deletion than in duplication carriers [12]. As for chromosome 16p13.11, also frequently detected in our study, the deletion was classified as pathogenic CNV, while duplication was classified as benign CNV with high frequency and low penetrance [13].

Despite the well-known clinical significance of many CNVs, some findings are often difficult to describe due to the absence of previous reports or different clinical findings from previous reports [7]. Among these, penetrance is a very careful variable to consider in genetic counselling. Despite 16p11.2 deletion and duplication being classified as LP, their penetrances are 62.4\%

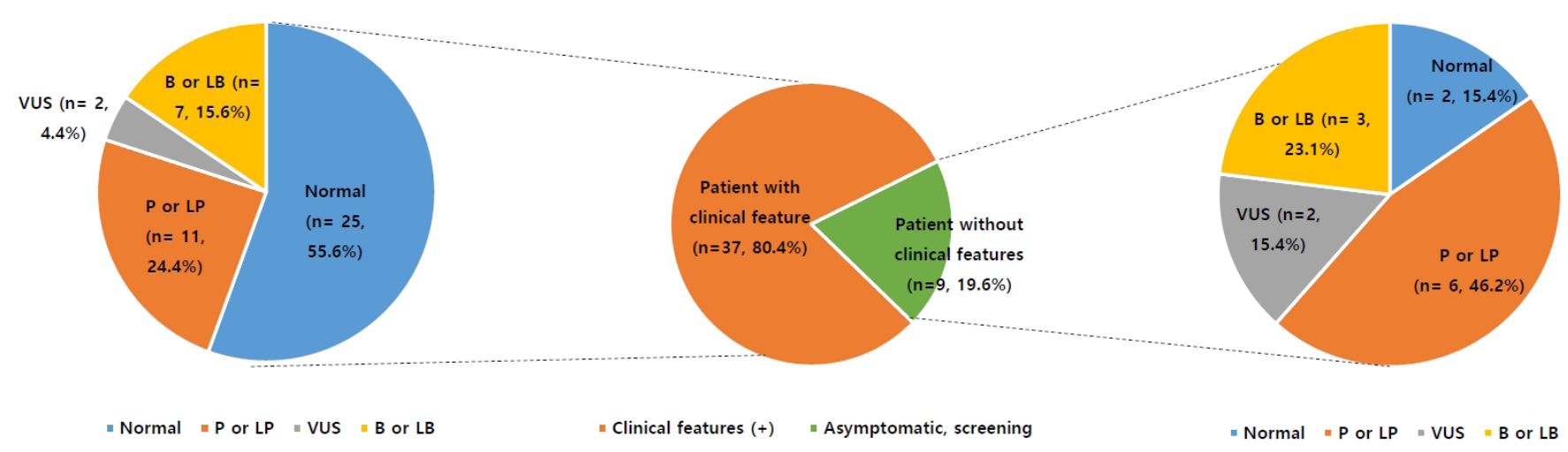

Fig. 1. The classification of CNVs detected in 46 patients requested chromosomal microarray analysis. Possible clinical significance variants including pathogenic (P), likely pathogenic (LP), and variant of unknowns significance (VUS) were $27.0 \%$ (10/37) in symptomatic patients and $77.8 \%$ (7/9) in asymptomatic or pre-symptomatic patients. B, benign; LB, likely benign. 
and $11.2 \%$ [14]. However, it is recommended that individuals had P/LP CNVs were regarding presymptomatic status [7]. Therefore, regular development and growth examination test is crucial and, furthermore, medical treatment should be determined through closely monitoring. On the other hand, for variations classified as VUS, there is a possibility of being classified as pathogenic in later, but this is a broad category classification that is more difficult to interpret. Generally they showed inconsistent evidence and it led to classification controversy among laboratories [7]. Many CNVs classified as VUS showed the genotype and phenotype correlation is not clear [8]. For example, the 18p11.31p11.2 duplication detected in asymptomatic patient A2 was classified as VUS, and confirmed as deriving from a healthy father. However, previous studies described that this variation is related to short stature, microcephaly and intellectual delay [15]. Hence, patients and family members should be periodically evaluated through genetic counseling.

In this study, we described CNV frequency with CMA and evaluated its clinical potential in a single tertiary hospital. The classification and interpretation of CMA remained a challenge. However, our study also showed that CMA is a superior method for CNV detection despite the limitation of the small sample size. Further research is needed to establish clinical diagnostic recommendations.

\section{Authors' Contributions}

Conception and design: HSP. Acquisition of data: HSP, KSS, BRS. Analysis and interpretation of data: HSP, AK. Drafting the article: HSP. Critical revision of the article: AK. Final approval of the version to be published: HSP.

\section{References}

1. Bi W, Borgan $C$, Pursley $A N$, Hixson $P$, Shaw $C A$, Bacino $C A$, et al. Comparison of chromosome analysis and chromosomal microarray analysis: what is the value of chromosome analysis in today's genomic array era? Genet Med 2013;15:450-7.

2. Freeman $\mathrm{J} L$, Perry GH, Feuk L, Redon R, McCarroll SA, Altshuler DM, et al. Copy number variation: new insights in genome diversity. Genome Res 2006;16:949-61.

3. Haraksingh RR, Abyzov A, Urban AE. Comprehensive performance comparison of high-resolution array platforms for genome-wide Copy Number Variation (CNV) analysis in humans. BMC Genomics
2017;18:321.

4. Riggs ER, Wain KE, Riethmaier D, Smith-Packard B, Faucett WA, Hoppman $N$, et al. Chromosomal microarray impacts clinical management. Clin Genet 2014;85:147-53.

5. Kaminsky EB, Kaul V, Paschall J, Church DM, Bunke B, Kunig D, et al. An evidence-based approach to establish the functional and clinical significance of copy number variants in intellectual and developmental disabilities. Genet Med 2011;13:777-84.

6. Zarrei M, MacDonald JR, Merico D, Scherer SW. A copy number variation map of the human genome. Nat Rev Genet 2015;16:172-83.

7. Riggs ER, Andersen EF, Cherry AM, Kantarci S, Kearney $H$, Patel $A$, et al. Technical standards for the interpretation and reporting of constitutional copy-number variants: a joint consensus recommendation of the American College of Medical Genetics and Genomics (ACMG) and the Clinical Genome Resource (ClinGen). Genet Med 2020;22:245-57.

8. Jang W, Kim Y, Han E, Park J, Chae H, Kwon A, et al. Chromosomal microarray analysis as a first-tier clinical diagnostic test in patients with developmental delay/intellectual disability, autism spectrum disorders, and multiple congenital anomalies: a prospective multicenter study in Korea. Ann Lab Med 2019;39:299-310.

9. Yao R, Yu T, Qing Y, Wang J, Shen Y. Evaluation of copy number variant detection from panel-based next-generation sequencing data. Mol Genet Genomic Med 2019;7:e00513.

10. Moreno-Cabrera JM, Del Valle J, Feliubadaló L, Pineda M, González S, Campos $\mathrm{O}$, et al. Screening of CNVs using NGS data improves mutation detection yield and decreases costs in genetic testing for hereditary cancer. J Med Genet 2020, in press.

11. Srour $M$, Shevell M. Genetics and the investigation of developmental delay/intellectual disability. Arch Dis Child 2014;99:386-9.

12. Steinman KJ, Spence SJ, Ramocki MB, Proud MB, Kessler SK, Marco EJ, et al.; Simons VIP Consortium. 16p11.2 Deletion and duplication: characterizing neurologic phenotypes in a large clinically ascertained cohort. Am J Med Genet A 2016;170:2943-55.

13. Kendall KM, Bracher-Smith M, Fitzpatrick $H$, Lynham $A$, Rees $E_{\text {, }}$ Escott-Price $V$, et al. Cognitive performance and functional outcomes of carriers of pathogenic copy number variants: analysis of the UK Biobank. Br J Psychiatry 2019;214:297-304.

14. Rosenfeld JA, Coe BP, Eichler EE, Cuckle H, Shaffer LG. Estimates of penetrance for recurrent pathogenic copy-number variations. Genet Med 2013;15:478-81.

15. Wang $R$, Zhang $H$, Li L, Yue F, Jiang $Y$, Li S, et al. Prenatal detection of interstitial 18p11.31-p11.22 microduplications: phenotypic diversity and literature review. Prenat Diagn 2019;39:1120-6. 